\title{
Correction to: Protection at First Sexual Intercourse Among Adolescent Girls and Young Women in Kenya
}

\author{
Edinah Casmir ${ }^{1}$ (D) Afkera Kesete Daniel ${ }^{2} \cdot$ Fernandos Ongolly $^{1} \cdot$ Nicholas Thuo $^{1} \cdot$ Lynda Oluoch $^{1}$. \\ Catherine Kiptinness ${ }^{1} \cdot$ Anna Wald $^{4,6,7,8} \cdot$ Nelly Rwamba Mugo $^{1,3} \cdot$ Alison C. Roxby $^{3,4}\left(\mathbb{C}^{\circ} \cdot\right.$ Kenneth Ngure $^{5}$
}

Published online: 4 September 2020

๑) Springer Science+Business Media, LLC, part of Springer Nature 2020

\section{Correction to:}

Archives of Sexual Behavior

https://doi.org/10.1007/s10508-020-01785-w

There was an error in this article as originally published.

The name of coauthor Afkera Kesete Daniel was given as Afkera Kasete.

The original article has been corrected.

Publisher's Note Springer Nature remains neutral with regard to jurisdictional claims in published maps and institutional affiliations.

The original article can be found online at https://doi.org/10.1007/ s10508-020-01785-w.

Edinah Casmir ednyab@gmail.com

1 Center for Clinical Research, Kenya Medical Research Institute, KNH, Nairobi 19865-0020, Kenya

2 Johns Hopkins University School of Medicine, Baltimore, MD, USA

3 Department of Global Health, University of Washington, Seattle, WA, USA

4 Department of Medicine, University of Washington, Seattle, WA, USA

5 Department of Community Health, Jomo Kenyatta University of Agriculture and Technology, Juja, Kenya

6 Department of Epidemiology, University of Washington, Seattle, WA, USA

7 Department of Laboratory Medicine, University of Washington, Seattle, WA, USA

8 Vaccines and Infectious Diseases Division, Fred Hutchinson Cancer Research Center, Seattle, WA, USA 